\title{
Synthesis and Study of Electrical Properties of SbTeI
}

\author{
Harish K. Dubey, ${ }^{1}$ L. P. Deshmukh, ${ }^{2}$ D. E. Kshirsagar, ${ }^{1}$ \\ Madhuri Sharon, ${ }^{3}$ and Maheshwar Sharon ${ }^{3}$ \\ ${ }^{1}$ Physics Department, Birla College, Kalyan 421301, India \\ ${ }^{2}$ Department of Physics, Solapur University, Solapur 413255, India \\ ${ }^{3}$ N.S.N. Research Center for Nanotechnology \& Bionanotechnology, Jambhul Phata, Ambernath (W) 421505, India
}

Correspondence should be addressed to Maheshwar Sharon; sharonmaheshwar@gmail.com

Received 19 August 2013; Revised 15 October 2013; Accepted 13 November 2013; Published 9 January 2014

Academic Editor: Taicheng An

\begin{abstract}
Copyright (C) 2014 Harish K. Dubey et al. This is an open access article distributed under the Creative Commons Attribution License, which permits unrestricted use, distribution, and reproduction in any medium, provided the original work is properly cited.

Needle shaped SbTeI crystals were obtained by solid state reaction. Electrical resistance was measured in the temperature range of $4 \mathrm{~K}$ to $550 \mathrm{~K}$. SbTeI shows a metallic behavior from $4 \mathrm{~K}$ to $300 \mathrm{~K}$, and at higher temperature $(>300 \mathrm{~K})$, it shows semiconducting behavior. Unlike SbSI, this material shows almost zero resistance around $550 \mathrm{~K}$. It shows a piezoelectric behavior with a capacitance of $717 \mathrm{pF}$ and its carrier density and nobilities are found to be $2.12 \times 10^{16} \mathrm{~cm}^{-3}$ and $1.01 \mathrm{~cm}^{2} /(\mathrm{V} \cdot \mathrm{s})$, respectively. Crystals of $\mathrm{SbTeI}$ are characterized by XRD, SEM, and Raman analysis. Electrical activation energy is found to be $0.52 \mathrm{eV}$. It is suggested that this material may be studied for its application as a superconductor with Tc higher than room temperature.
\end{abstract}

\section{Introduction}

Many ternary chalcogenide compounds with the combination of $\mathrm{Sb}, \mathrm{S}, \mathrm{Te}, \mathrm{Bi}, \mathrm{Cl}, \mathrm{Br}, \mathrm{Se}$, and $\mathrm{I}$ have been reported in the recent years and various attempts have been made to study their applications, like the electrostrictive constant, electroceramics property, semiconducting properties $[1,2]$, and so forth. SbSI is one of the most intensively studied compounds of this family [3-6]. Nitsche and Merz [7] have synthesized materials like SbSI, SbSBr, SbSeBr, SbSeI, SbTeI, $\mathrm{BiSCl}, \mathrm{BiSBr}, \mathrm{BiSI}, \mathrm{BiSeCl}$, and $\mathrm{BiSeBr}$ and studied their photoconducting properties. Papazoglou and Rentzeperis [8] have examined in detail the crystal structure of SbTeI and also reported that SbSI has metallic and semiconducting behavior. Kichambare et al. [9-11] prepared SbTeI at different temperatures and calculated the values of lattice constants, ionization potentials, and activation energies. Later, they doped SbTeI with Bi and studied its impact on the values of lattice constant, ionization potential, and activation energy.

Since in-depth studies on the electrical properties of SbTeI are not made [3-6, 8-12], it was thought to be desirable to study the electrical properties of SbTeI to find out the carrier concentration, mobility, piezoelectric property, capacitance, and so forth. In this paper, we report synthesis of SbTeI using solid state reaction technique and electrical properties studies using van der Pauw and two probe techniques in the temperature range of $4 \mathrm{~K}-550 \mathrm{~K}$.

\section{Experimental}

2.1. Synthesis of SbTeI by Solid State Thermal Reaction. SbTeI was synthesized by keeping equal weighed quantity of the constituents (Se, Te and I) in a quartz tube of $24 \mathrm{~cm}$ length and $1.0 \mathrm{~cm}$ diameter (Figure $1(\mathrm{a})$ ). The quantities of $\mathrm{Sb}, \mathrm{Te}$, and I were taken in the ratio $1: 1: 1$, that is, 2 gm each. The tube was sealed under vacuum and then kept in a PID controlled tubular furnace heated to a set temperature (Figure 1(b)). Based on several experiments, finally two temperatures $400^{\circ} \mathrm{C}$ and $600^{\circ} \mathrm{C}$ were selected for the synthesis of SbTeI. After the end of thermal treatment the furnace was cooled down slowly at room temperature. One end of the tube was broken to take out the material. Crystals were deposited on the walls of the quartz tube. Needle shaped crystals were formed (Figure 1(c)). The reaction involved in the process is

$$
\mathrm{Sb}+\mathrm{Te}+\mathrm{I}=\mathrm{SbTeI} .
$$




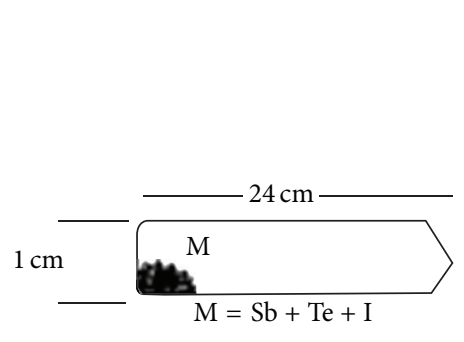

(a)

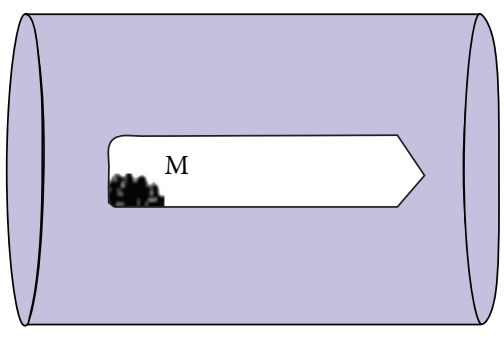

(b)

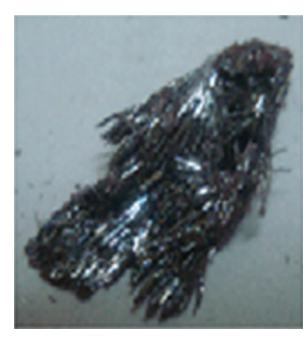

(c)

Figure 1: (a) Quartz tube of length $24 \mathrm{~cm}$ and diam. 1 $\mathrm{cm}$ containing mixture of Sb, Te, and I in equal portion. (b) Quartz tube (M) kept in a tubular furnace and (c) photograph of needle shaped SbTeI.

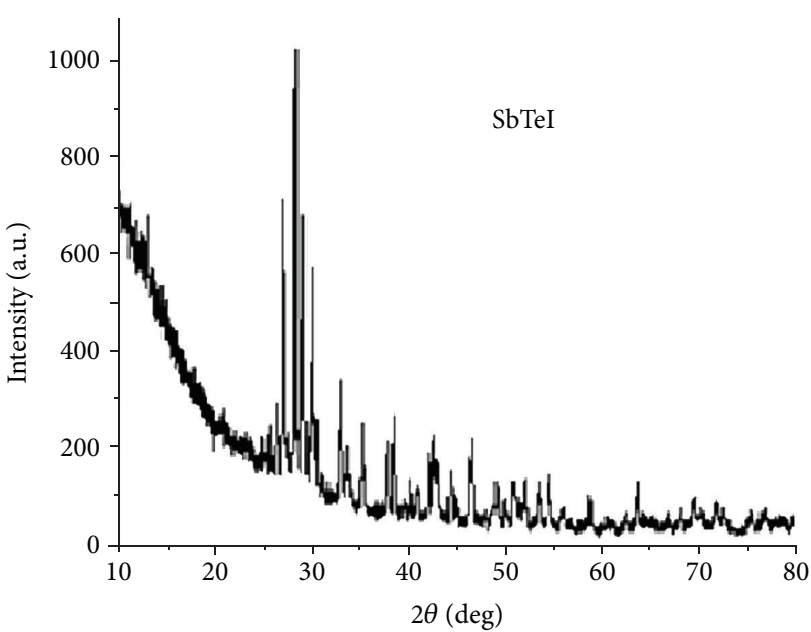

(a)

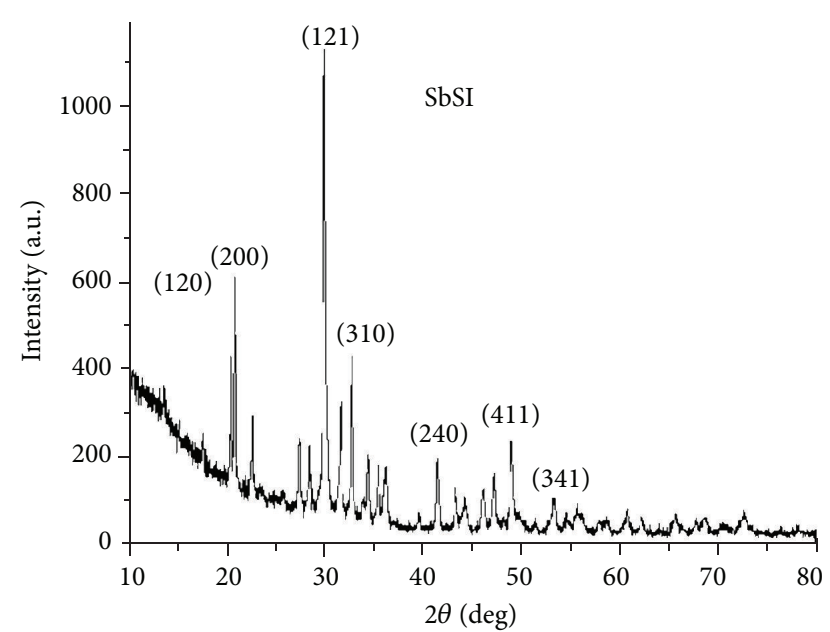

(b)

FIgURE 2: X-ray diffractogram of a (a) SbTeI and (b) SbSI.

2.2. The X-Ray Diffraction (XRD) Study of SbTeI. The XRD pattern was recorded by a Phillips PW 3710 X-ray diffractometer using $\mathrm{CuK} \alpha$ radiation. The scanning angle $(\theta)$ was set between $10^{\circ}$ and $80^{\circ}$. The XRD patterns of the powdered samples were taken (Figure 2).

2.3. The Scanning Electron Microscopy (SEM) of SbTeI. SEM images of two typical samples of SbTeI were taken using JEOL-6360 (LA) EDAX cum SEM. Probe current of $1 \mathrm{nA}$ was set in the energy range of $0-20 \mathrm{KV}$ for scanning the samples (Figure 3).

2.4. Micro-Raman Spectroscopy of SbTeI-HORIBA. JobinYvon Lab Raman spectrophotometer was utilized to obtain Raman spectra. Based on the Helium-Neon laser source $(632.88 \mathrm{~nm})$, laser pulses were generated with the resolution $<1.5 \mathrm{~cm}^{-1}$. All measurements were performed using a $50 \mathrm{X}$ objective lens and D 0.3 filter randomly at 10 different positions (Figure 4).

2.5. Electrical Resistivity of SbTeI. Since the crystals were not of very large size, the crystals were powdered with a hand grinder and pressed with $140 \mathrm{Kg} / \mathrm{cm}^{3}$ pressure with a 10 ton hydraulic press to make a pellet. The pellets were circular with $1 \mathrm{~cm}$ diameter and 0.5 to $1.5 \mathrm{~mm}$ thickness. Pellets were made without the use of any binder. To make the pellets strong, they were sintered for $90 \mathrm{~min}$ at $200^{\circ} \mathrm{C}$. Pellets of SbTeI were used to study the electrical (Figure 5) and dielectric properties. Many pellets were used for these studies to confirm their reproducibility.

2.6. Charge Carrier Density Determination of SbTeI. The charge carrier density was determined by Hall Effect measurement with the Polytronics Hall apparatus set-up. At constant magnetic field of 2790 Gauss, applied current was varied and the corresponding voltages were measured to calculate the Hall Coefficients. Average of all values was used to calculate donor density.

2.7. Dielectric Constant of SbTeI. The dielectric constant was calculated by sandwiching pellet of SbTeI between two parallel plates of PCB sheet of size $1 \times 1.2 \mathrm{~cm}$. The surface of copper coating was exposed to the material. Two copper leads were soldered to these plates. LCR meter (Aplab India) was used for this purpose. The capacitance was measured at $1 \mathrm{KHz}$ and $10 \mathrm{KHz}$ frequencies. It was found that the capacitance in both the frequencies was almost the same. Therefore, 

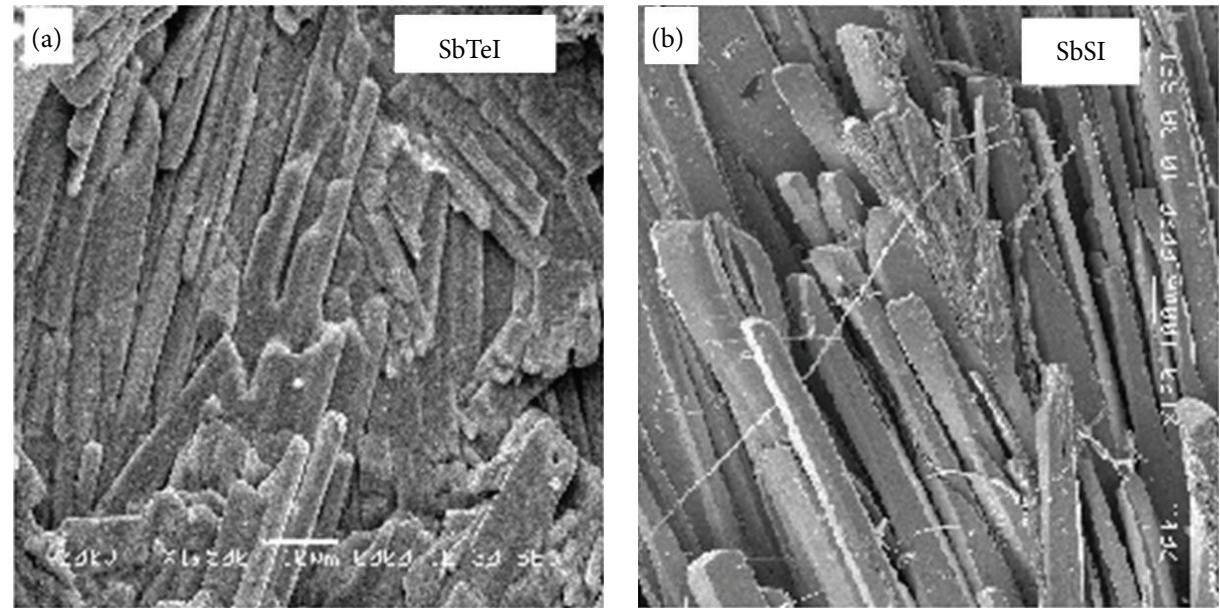

Figure 3: SEM micrographs (a) SbTeI and (b) SbSI.

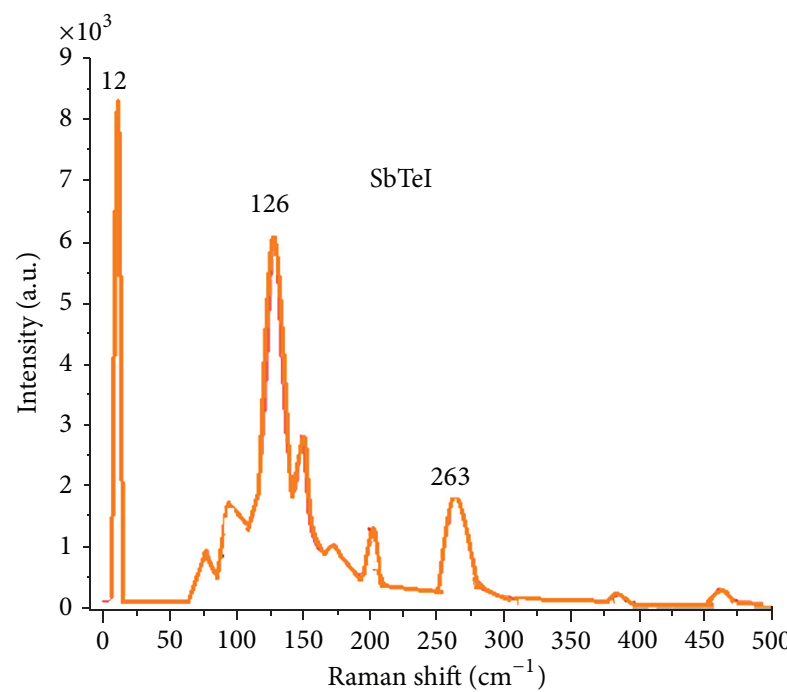

(a)

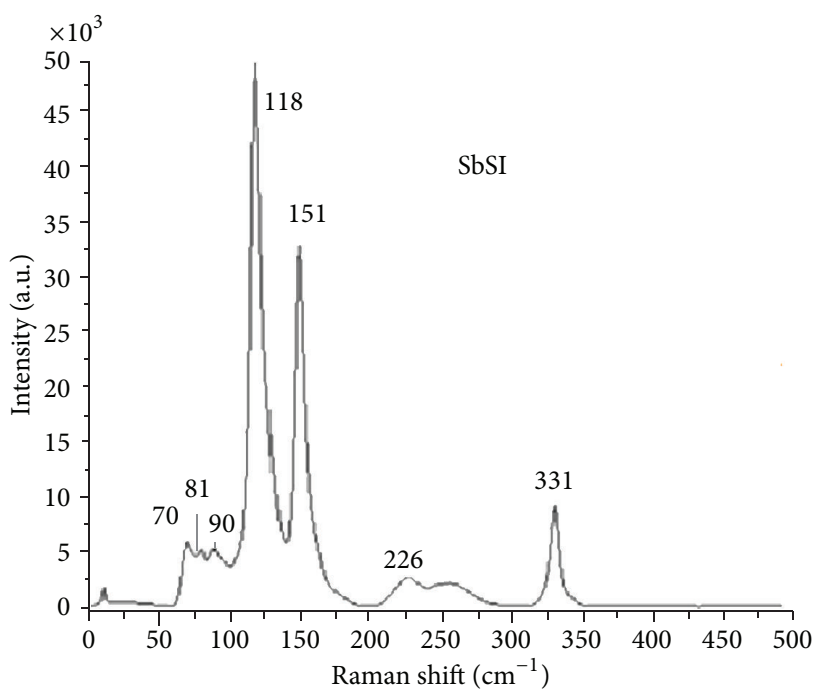

(b)

Figure 4: Micro-Raman plot for (a) SbTeI-2 and (b) SbSI.

the calculations were done on the basis of the capacitance observed for $1 \mathrm{KHz}$.

2.8. Piezoelectric Behavior of SbTeI. The system used for measuring the dielectric constant was also used to study the ohmic behavior of SbTeI.

\section{Results and Discussions}

3.1. XRD Analysis. XRD peaks obtained with SbTeI are shown in Figure 2(a). Due to nonavailability of JCPDS data, indexing of SbTeI was not possible. However, for comparison, the XRD of SbSI is shown in Figure 2(b) [13]. It is observed that two peaks around 20 degree observed with SbSI are absent with SbTeI. In addition, several closely packed peaks at an angle $<30$ degree were observed with SbTeI. This is in contrast to peak observed with SbSI. Though they are similar, they are well separated at $>30$ degree (Figure 2(b)) [13]. Apart from these variations, other low intensities peaks at angle greater than 40 degree are also observed which are of similar nature for both materials.

3.2. SEM Image Observations. Figure 3(a) shows the SEM images of SbTeI and Figure 3(b) shows the SEM of SbSI [13]. It is observed that though both show the rod type structures, SbTeI shows very compact branched type rods whereas rods of SbSI are separated [13]. In Table 1, SEM micrographs of two selected samples of SbTeI are shown. Enlarged micrographs show the rod to be hollow. SEM micrographs suggest that long duration $(480 \mathrm{~h})$ of thermal treatments at $400^{\circ} \mathrm{C}$ produces better crystal structure than sample treated for shorter duration $(4 \mathrm{~h})$ at higher temperature $\left(600^{\circ} \mathrm{C}\right)$. From the SEM images of the samples it is confirmed that SbTeI grew in rod shaped orthorhombic crystals. This is also in agreement with the previously reported results [14-16]. 


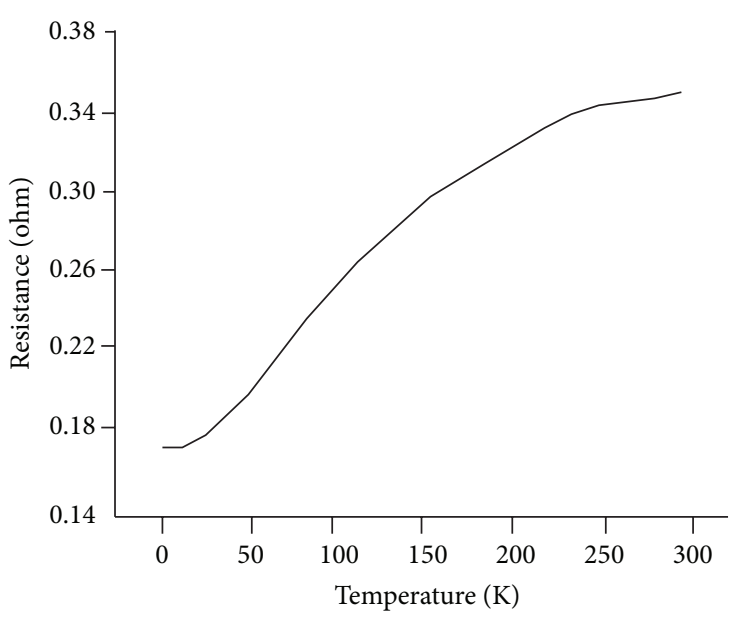

(a)

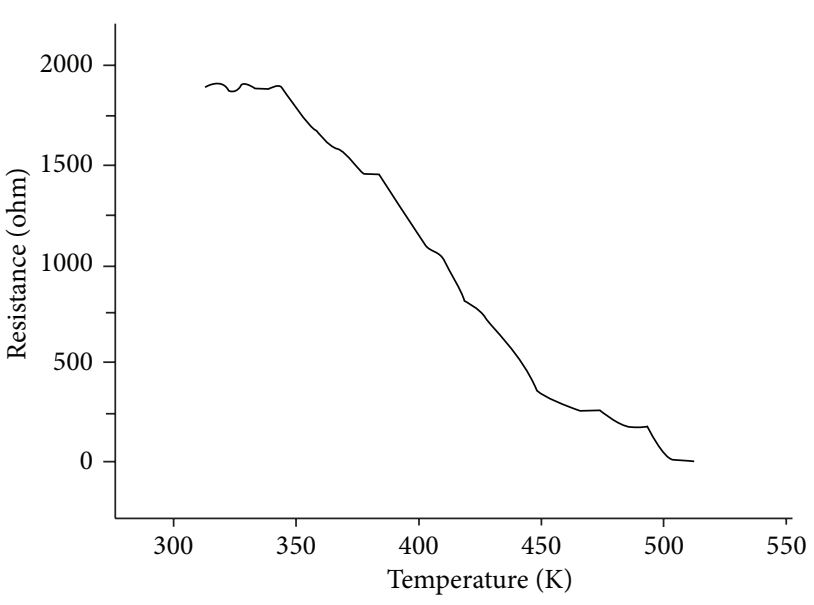

(b)

FIGURE 5: (a) Graph showing the variation in resistance versus temperature of SbTeI sample in the temperature range of $4 \mathrm{~K}$ to $300 \mathrm{~K}$ and (b) variation in resistance versus temperature of SbTeI in the temperature range $313 \mathrm{~K}$ to $523 \mathrm{~K}$.

TABLE 1: SEM morphologies of compounds prepared under different conditions.

\begin{tabular}{|c|c|c|c|}
\hline Sample & SbTeI Growth conditions & SEM images of SbTeI Crystals & Crystal details \\
\hline SbTeI-1 & $\begin{array}{c}\text { Thermal treatment } \\
\text { at } 400^{\circ} \mathrm{C} \\
\text { for } 480 \mathrm{hrs}\end{array}$ & & $\begin{array}{c}\text { Size } 2-8 \mu \mathrm{m} \text {. } \\
\text { Smooth rods stacked in random } \\
\text { fashion. } \\
\text { Enlarged micrograph shows } \\
\text { hollow rods }\end{array}$ \\
\hline SbTeI-2 & $\begin{array}{l}\text { Thermal treatment } \\
\text { at } 600^{\circ} \mathrm{C} \\
\text { for } 4 \mathrm{hrs}\end{array}$ & & $\begin{array}{c}\text { Size } 5-20 \mu \mathrm{m} \text {. } \\
\text { Mixture of rods, rectangular } \\
\text { plates and, big plates }\end{array}$ \\
\hline
\end{tabular}

TABLE 2: Stoichiometric composition of SbTeI calculated from EDAX results.

\begin{tabular}{lc}
\hline Sample and synthesis conditions & \% elemental composition \\
\hline SbTeI- 1 & $\mathrm{Sb}=37.57$ \\
Time-480 hrs & $\mathrm{Te}=34.11$ \\
Temp-400 $\mathrm{C}$ & $\mathrm{I}=27.41$ \\
SbTeI-2 & $\mathrm{Sb}=31.63$ \\
Time-4hrs & $\mathrm{Te}=27.57$ \\
Temp- $600^{\circ} \mathrm{C}$ & $\mathrm{I}=40.80$ \\
\hline
\end{tabular}

3.3. Micro-Raman Assessment of SbTeI. The peaks observed in Raman spectrum of SbTeI is shown in Figure 4(a). For comparison, Raman spectrum of SbSI is shown in Figure 4(b)
[13]. It is noticed that most of peaks obtained with SbTeI appear at different Raman shift as compared to Raman spectrum of SbSI except one peak at $12 \mathrm{~cm}^{-1}$, which is absent in SbSI.

3.4. EDAX Analysis. Stoichiomentric composition of SbTeI was calculated from the EDAX attached to the SEM unit. Composition of two samples of SbTeI is presented in Table 2. It is noticed that $\mathrm{SbTeI}$ synthesized at $400^{\circ} \mathrm{C}$ for $480 \mathrm{~h}$ shows deficiency in iodine while sample prepared at $600^{\circ} \mathrm{C}$ for $4 \mathrm{~h}$ shows deficiency in $\mathrm{Te}$ and excess in iodine, assuming the composition to be $(1: 1: 1)$. It can also be concluded from these results that SbTeI prepared at $400^{\circ} \mathrm{C}$ for $480 \mathrm{~h}$ contains 


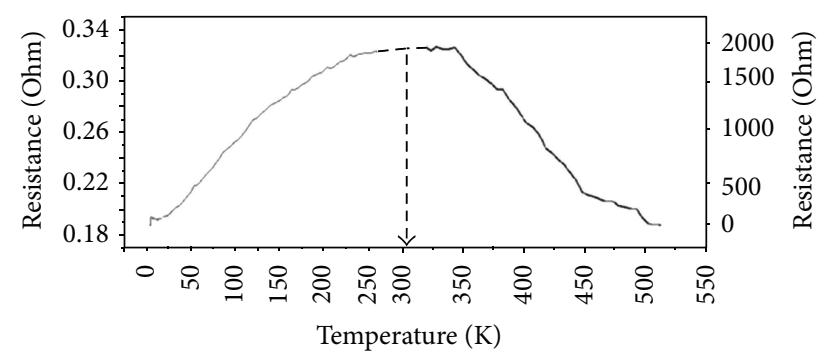

FIgURE 6: Graphs shown in Figures 5(a) and 5(b) for SbTeI are combined to examine the variation in resistance from $4 \mathrm{~K}$ to $550 \mathrm{~K}$.

composition nearer to $1: 1: 1$ as compared to sample prepared at $600^{\circ} \mathrm{C}$.

3.5. Electrical Resistivity Measurements. Resistance of SbTeI was measured by four probe method in temperature range $4 \mathrm{~K}$ to $300 \mathrm{~K}$. Variation of voltage of SbTeI sample at different temperatures was recorded at constant current of $100 \mathrm{~mA}$. Resistance was calculated for each of measurements. The variation in resistance with temperature is plotted (Figure 5(a)). The trend in the resistance behavior at different temperatures confirms SbTeI to behave like metal.

By using two probe technique, resistance of SbTeI was measured in the temperature range of $300 \mathrm{~K}$ to $550 \mathrm{~K}$. Results are plotted in Figure 5(b). This graph suggests that SbTeI behaves like a semiconductor from $300 \mathrm{~K}$ to higher temperature. In order to observe the transition temperature when SbTeI shifts its behavior from metallic to semiconducting, both of these graphs were joined and plotted (Figure 6).

It can be noticed from Figure 6 that the resistance increased with increase of temperature from $4 \mathrm{~K}$ to $250 \mathrm{~K}$ and from around $300 \mathrm{~K}$, it starts decreasing linearly with increase of temperature. It is also interesting to note that, while resistance of SbSI was observed to be in Mega ohm [13] even at around $500 \mathrm{~K}$, resistance of SbTeI tends towards zero resistance at higher temperature. This is a unique property of SbTeI and needs to carry out research to confirm whether it can show a superconductivity behavior at such a high temperature. If this is possible, it would be a result of great interest.

3.6. Activation Energy for the Conduction. The resistivity $(\rho)$ of SbTeI sample was calculated at different temperature in the range of $313 \mathrm{~K}$ to $523 \mathrm{~K}$. Finally, a graph of $\ln (\rho)$ versus $(1 / T) 10^{3}$ was plotted (Figure 7$)$.

From the graph (Figure 7) of $\ln (\rho)$ versus $(1 / T) 10^{-3}$, the activation energy for the conduction was calculated by measuring the slope of the linear portion of the plot in the higher temperature region. The slope was found to be 3.055 $\times 10^{-3} \mathrm{~K}$ from which the activation energy was found to be $0.52 \mathrm{eV}$.

3.7. Charge Carrier Density. Carrier density was calculated by Hall measurement technique. The result of Hall voltage

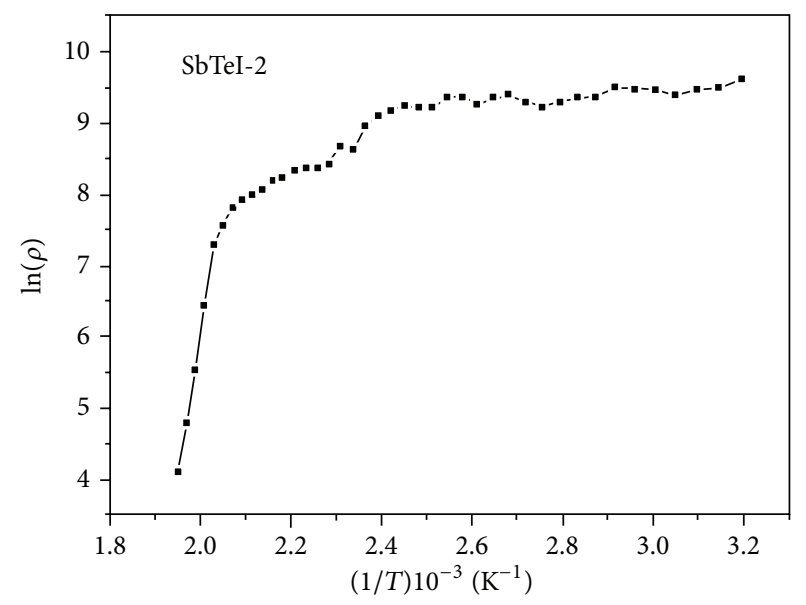

Figure $7: \ln (\rho)$ versus $(1 / T) 10^{-3} \mathrm{~K}$ graph of SbTeI sample.

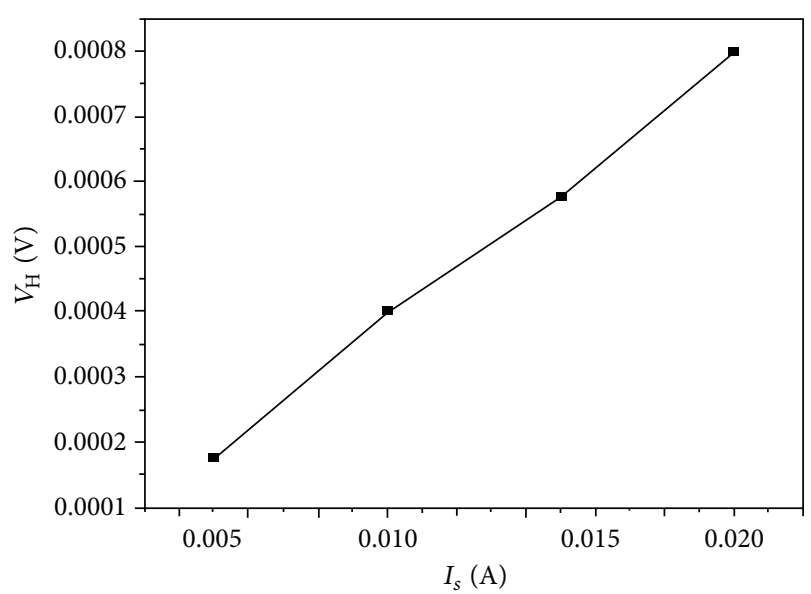

Figure 8: Graph of $V_{H}$ versus $I_{s}$ for SbTeI sample.

$\left(V_{H}\right)$ versus current $\left(I_{s}\right)$ was plotted (Figure 8) which gives a linear graph. From the slope of the graph, Hall coefficient $R_{H}$ was calculated 293.90 . Using this value, the carrier density and mobility of carriers were calculated $2.12 \times 10^{16} \mathrm{~cm}^{-3}$ and $1.01 \mathrm{~cm}^{2}(\mathrm{~V} \cdot \mathrm{s})$, respectively.

3.8. Dielectric Constant. To avoid the impact of the stray capacitance, the capacitance of the empty capacitor with the spacing between the plates equal to the thickness of the sample was subtracted from the capacitance with the sample. The net capacitance of SbSI sample was found to be $717 \mathrm{pF}$.

Using the relation $k=(C \times d) /\left(A \times 8.854 \times 10^{-12}\right)$, the value of $k$ is found to be 10112 .

3.9. Piezoelectric Behavior of SbTeI. The SbTeI sample was found to be ohmic (Figure 9) in nature. It was interesting to observe that SbTeI pellet shows significant decrease in the resistance when pressed between two plates; that is, resistance was found to decreased from 100's of ohms to as low as 10 ohms. 


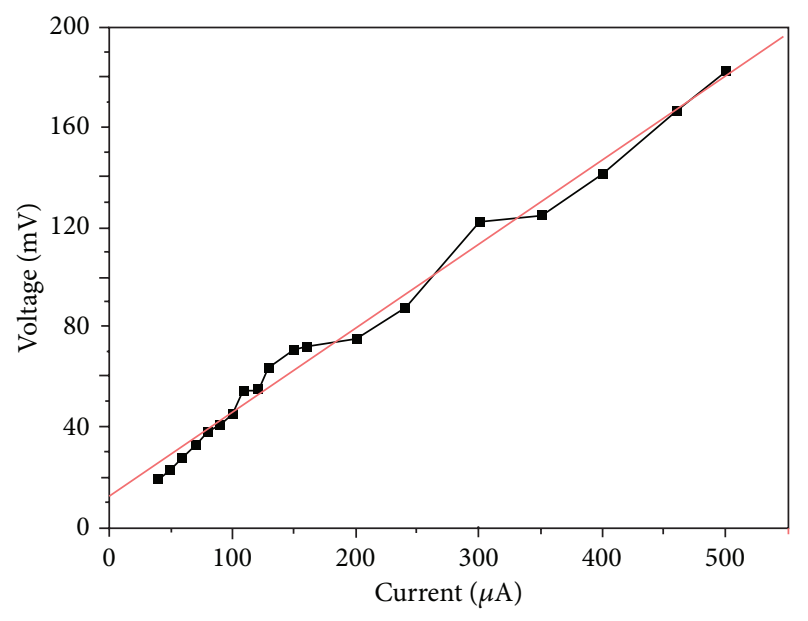

Figure 9: $I-V$ graph of SbTeI at room temperature when pressed between two plates.

This indicates the piezoelectric property of SbTeI. As the material belongs to SbSI family and SbSI is already confirmed to be a piezoelectric material [8], there is a strong possibility that $\mathrm{SbTeI}$ is also a piezoelectric material.

\section{Conclusions}

SbTeI was synthesized by solid state reaction. SEM micrographs show that SbTeI crystals are rod shaped. SbTeI shows metallic conductivity in the temperature range $4 \mathrm{~K}$ to $\sim 300 \mathrm{~K}$ and semiconducting bahaviour in temperature higher than $300 \mathrm{~K}$. The resistance of SbTeI tends to almost zero at around $550 \mathrm{~K}$. Its mobility and carrier density are calculated to be 2.12 $\times 10^{16} \mathrm{~cm}^{-3}$ and $1.01 \mathrm{~cm}^{2} /(\mathrm{V} \cdot \mathrm{s})$, respectively. It is proposed that $\mathrm{SbTeI}$ is a piezoelectric material. The activation energy obtained by electrical measurement in the range of $300 \mathrm{~K}$ to $550 \mathrm{~K}$ is $0.52 \mathrm{eV}$. The electrical properties of $\mathrm{SbTeI}$ are reported for the first time. SbTeI is characterized by XRD, SEM, and Raman analysis. More study is needed to confirm whether this material can be used as superconductor.

\section{Conflict of Interests}

The authors declare that there is no conflict of interests regarding the publication of this paper.

\section{References}

[1] M. Nowak, P. Mroczek, P. Duka et al., "Using of textured polycrystalline SbSI in actuators," Sensors and Actuators A, vol. 150, no. 2, pp. 251-256, 2009.

[2] A. Audzijonis, L. Žigas, I. V. Vinokurova et al., "Investigation of the vibration spectrum of SbSI crystals in harmonic and in anharmonic aproximations," Physica B, vol. 373, no. 1, pp. 157$162,2006$.

[3] A. Starczewska, M. Nowak, P. Szperlich et al., "Influence of humidity on impedance of SbSI gel," Sensors and Actuators A, vol. 183, pp. 34-42, 2012.
[4] J. Grigas, A. Kajokas, A. Audzijonis, and L. Žigas, "Peculiarities and properties of SbSI electroceramics," Journal of the European Ceramic Society, vol. 21, no. 10-11, pp. 1337-1340, 2001.

[5] I. Cho, B. K. Min, S. W. Joo, and Y. Sohn, "One-dimensional single crystalline antimony sulfur iodide, SbSI," Materials Letters, vol. 86, pp. 132-135, 2012.

[6] A. Audzijonis, R. Žaltauskas, L. Žigas et al., "Variation of the energy gap of the SbSI crystals at ferroelectric phase transition," Physica B, vol. 371, no. 1, pp. 68-73, 2006.

[7] R. Nitsche and W. J. Merz, "Photoconduction in ternary V-VIVII compounds," Journal of Physics and Chemistry of Solids, vol. 13, no. 1-2, pp. 154-155, 1960.

[8] A. G. Papazoglou and P. J. Rentzeperis, "The crystal structure of antimony telluroiodide, SbTeI," Zeitschrift für Kristallographie, vol. 165, no. 1-4, pp. 159-167, 1983.

[9] P. D. Kichambare and M. Sharon, "Synthesis, structure and conductivity of new quaternary chalcogenide SnBi4Te4Se4," Solid State Ionics, vol. 62, no. 1-2, pp. 21-26, 1993.

[10] P. Kichambare and M. Sharon, "Preparation, characterization and physical properties of mixed Sb1-xBixTeI," Solid State Ionics, vol. 101, no. 1, pp. 155-159, 1997.

[11] P. Kichambare, M. Sharon, Y. Seki, T. Hagino, and S. Nagata, "Preparation and fundamental physical properties of the quaternary chalcogenides SnBi4-xTe3+ySe4-z and PbBi4-xTe3ySe4-z," Solid State Ionics, vol. 101, no. 1, pp. 125-129, 1997.

[12] Z. S. Aliev, M. B. Babanly, A. V. Shevelkov, D. M. Babanly, and J.-C. Tedenac, "Phase diagram of the Sb-Te-I system and thermodynamic properties of SbTeI," International Journal of Materials Research, vol. 103, no. 3, pp. 290-295, 2012.

[13] K. D. Harish, L. P. Deshmukh, D. E. Kshirsagar, M. Sharon, and M. Sharon, "A study of the electrical properties of SbSI synthesized using CVD techniques," QScience Connect, vol. 2013, article 40, 2013.

[14] Z. Dziuba, "Crystallization of SbSI crystal from the vapour phase," Journal of Crystal Growth, vol. 35, no. 3, pp. 340-342, 1976.

[15] G. Raman, F. D. Gnanam, and P. Ramasamy, "Growth of antimony sulphoiodide in gel," Journal of Crystal Growth, vol. 69, no. 2-3, pp. 404-406, 1984.

[16] K. Nassau, J. W. Shiever, and M. Kowalchik, "The growth of large SbSI crystals: control of needle morphology," Journal of Crystal Growth, vol. 7, no. 2, pp. 237-245, 1970. 

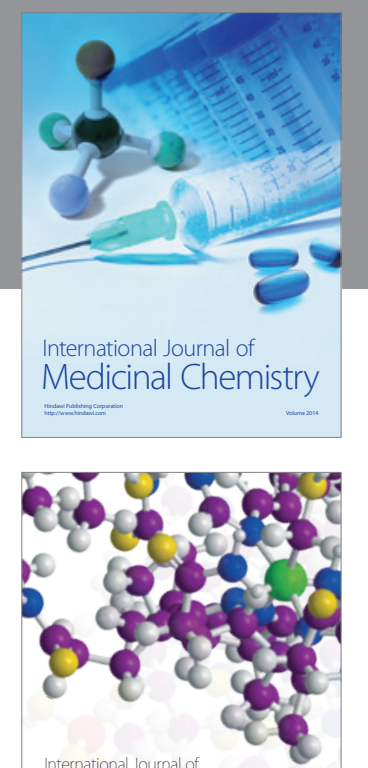

\section{Carbohydrate} Chemistry

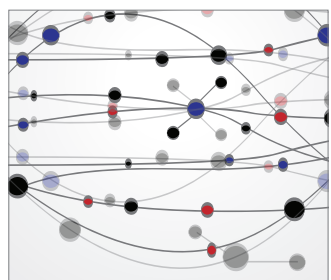

The Scientific World Journal
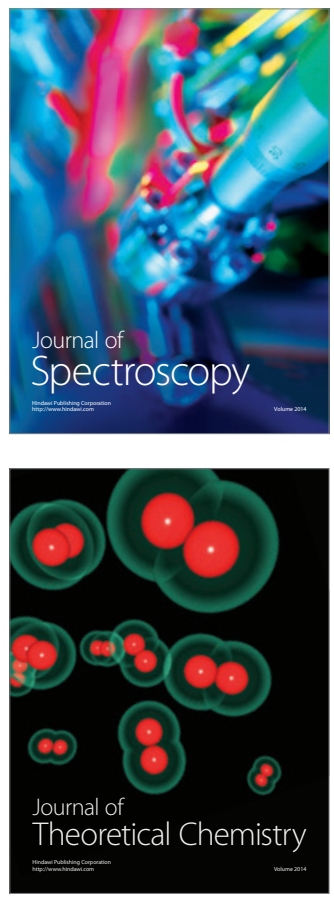
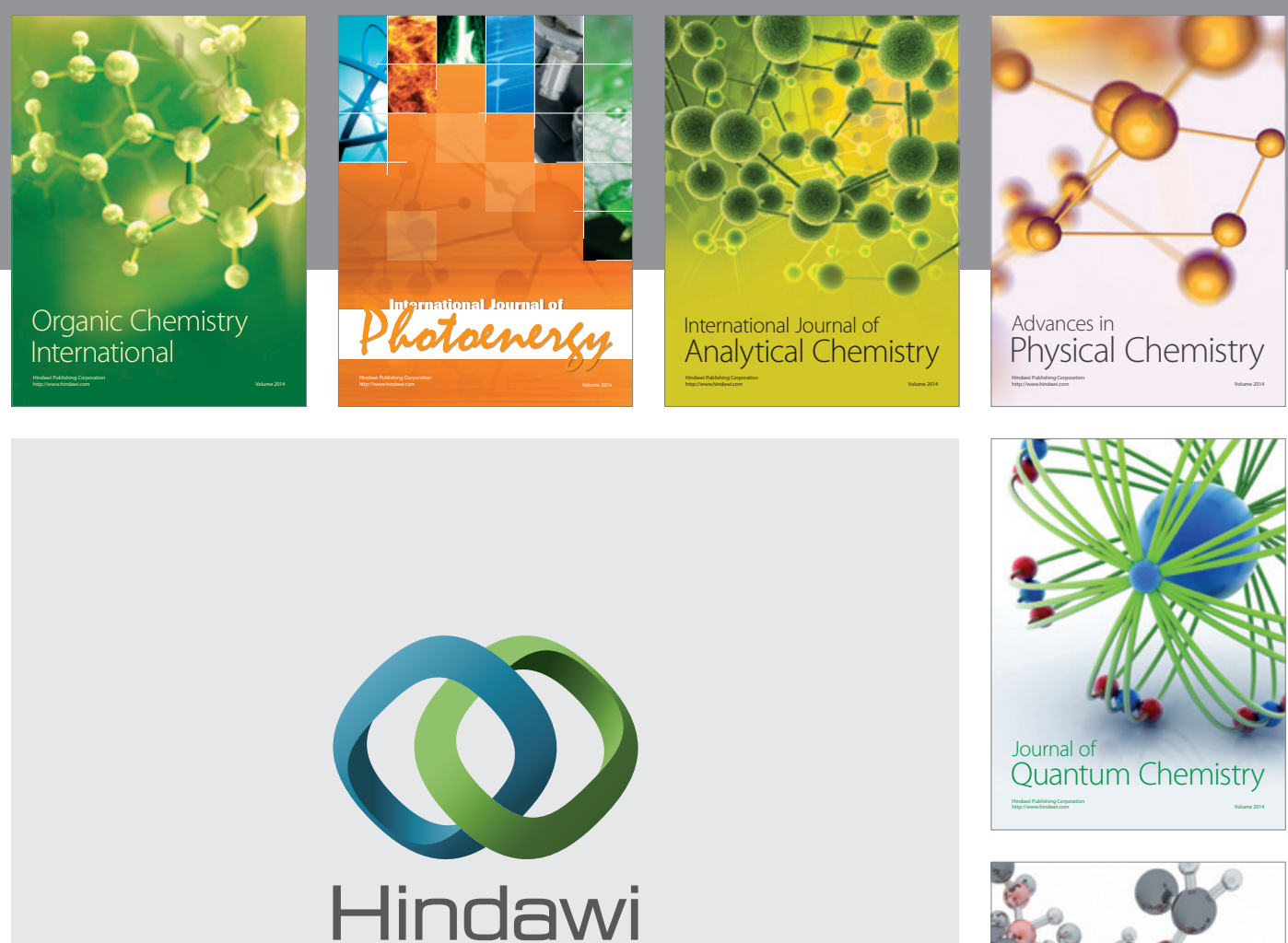

Submit your manuscripts at

http://www.hindawi.com

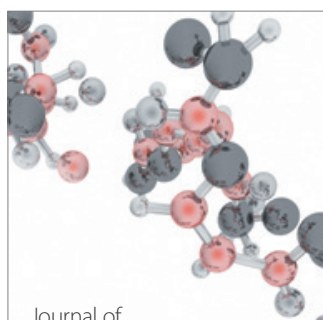

Analytical Methods

in Chemistry

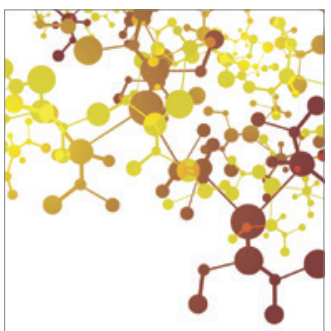

Journal of

Applied Chemistry

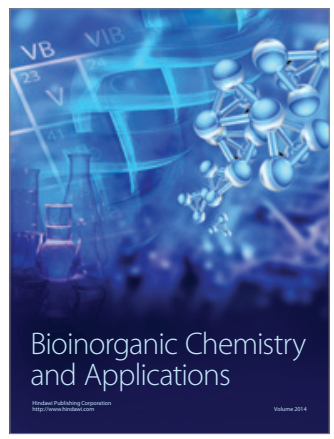

Inorganic Chemistry
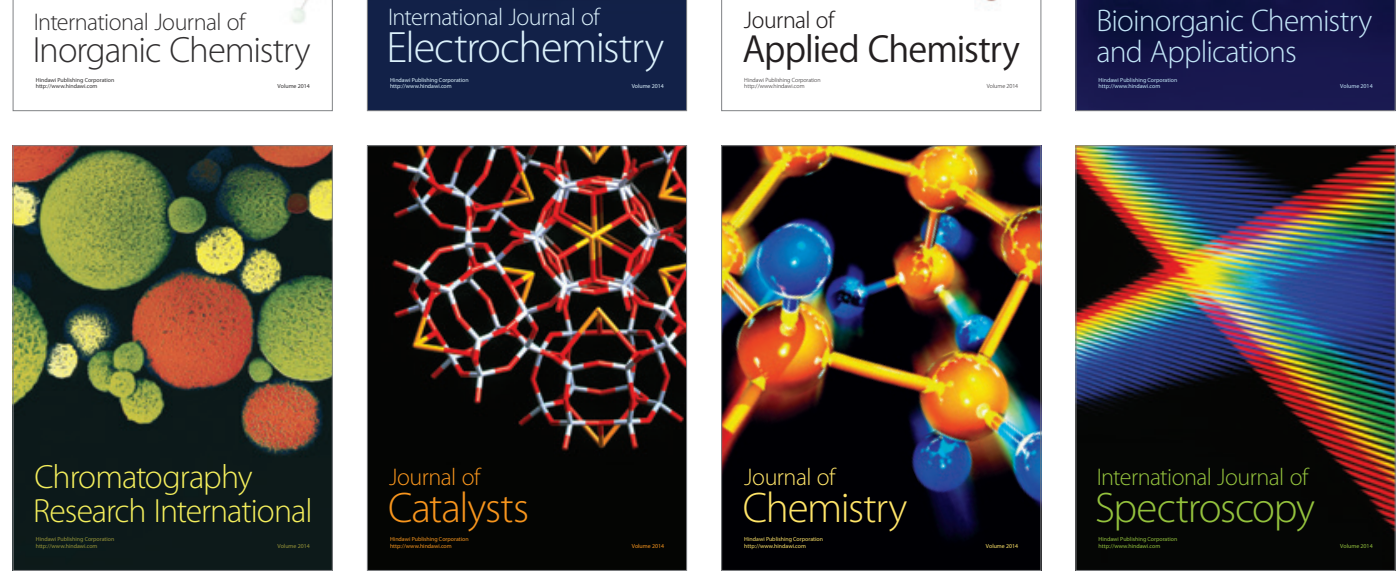